\title{
Endorsement of the HerniaSurge guidelines by the Afro Middle East Hernia Society
}

\author{
R. Sani ${ }^{1}$
}

Received: 13 July 2017 / Accepted: 20 September 2017 / Published online: 12 January 2018

c) Springer-Verlag France SAS 2017

Congratulations to the HerniaSurge group for this huge job. The Afro Middle East Hernia Society is the youngest/most recent among the biggest abdominal wall surgery societies. Hernia constitutes a real health issue in Africa and Middle East. The elaboration of guidelines was for AMEHS, one of the first, major activities within the HerniaSurge group. Indeed, these guidelines will allow surgeons from low resource countries to adequately and correctly address hernia pathology. To do this job within the HerniaSurge group, AMEHS designated Prof KONATE IBRAHIMA, President of Senegalese Hernia Society (SHS) and myself, President of AMEHS, from NIGER to be part of this important work. We were involved at all stages such as the multiple e-mail exchanges and the World Congress held in Milan. We also took part in the voting of these guidelines during the EHS Congress in Rotterdam. The dynamism of Maarten Simons and of all members of other societies resulted into putting in place these guidelines. AMEHS's members participated in voting in favor of these guidelines, either live for those who were present in Rotterdam or via internet for the others. People were most satisfied because these guidelines took into account their concerns.

Members of AMEHS kindly request to be involved in such scientific events/activities, especially because hernia surgery represents 1 out of every 2 abdominal surgeries. I am taking this opportunity to call on firms who make meshes, to consider selling to sub-Saharan African countries which are the future in the use of meshes. Today, in many countries, African orthopedic surgeons solved this issue for hip and knee surgery by using prosthesis. So why not abdominal surgeons?

AMEHS had the pleasure of working actively in this project and now endorses these guidelines and plans to participate in other studies within the HerniaSurge group.

Prof. Rachid Sani, President AMEHS.

\section{Compliance with ethical standards}

Conflict of interest The author declares that he has no conflict of interest.

This comment refers to the article available at doi:10.1007/ s10029-017-1668-x.

R. Sani

sani.rachid@gmail.com

1 Department of Surgery, University of Niamey, Niamey, Niger 